\title{
Fluorescence biosensor based on encapsulated quantum dots/enzymes/sol-gel for non-invasive detection of uric acid
}

\begin{abstract}
Non-invasive and simple method provides an alternative tool for the detection of uric acid in human biological sample without drawing a blood or puncturing the skin. This present work report a novel fluorescence biosensor based on sol-gel encapsulated $\mathrm{CdS}$ quantum dots (QDs)-Uricase/Horseradish Peroxidase (HRP) enzymes in 96-well microplate format. The QDs is used as fluorescence indicator to reveal fluorescence signal of the system resulting from the enzymatic reaction of uricase/HRP in the presence of uric acid. Upon addition of uric acid to the hybrid QDs-uricase/HRP, it will be oxidized to yield allaintoin, $\mathrm{CO} 2$ and $\mathrm{H} 2 \mathrm{O} 2$. The produced $\mathrm{H} 2 \mathrm{O} 2$ has the ability to quench the QDs fluorescence intensity which is proportional to the uric acid concentration. The developed microplate biosensor has shown its advantage in term of good system for simultaneously detection of 96 samples per assay within $20 \mathrm{~min}$. The linear calibration curve towards uric acid was in the concentration range of 60 $2000 \mu \mathrm{M}$ with the detection limit of $50 \mu \mathrm{M}$. The developed sensor has successfully applied for the detection of uric acid in human urine and the results was comparable with the assay kit.
\end{abstract}

Keyword: Non-invasive; Quantum dots; Uric acid; Biosensor; Fluorescence 
